\title{
CRITÉRIOS MASCULINOS E FEMININOS DE ESCOLHA DE PARCEIROS EM UMA AMOSTRA DE ESTUDANTES UNIVERSITÁRIOS DA CIDADE DE RIO VERDE-GO
}

Jannaine Calixto Macedo Guimarães, Universidade de Rio Verde, janainneguimaraes@hotmail.com. Claudio Herbert Nina-e-Silva, Universidade de Rio Verde.

\section{Recebido em: 28/05/2013 - Aprovado em: 15/07/2013 - Disponibilizado em: 15/08/2013}

Resumo: O presente estudo objetivou realizar uma comparação entre critérios masculinos e femininos de escolha de parceiros. Participaram 50 homens e 50 mulheres, estudantes da Universidade de Rio Verde, que responderam a um questionário referente a dados pessoais e às características de um hipotético parceiro para relacionamento afetivo duradouro. Não houve diferenças estatisticamente significativas entre os gêneros na atribuição de importância às características de potenciais parceiros ( $t=1,24 ; p=0,12$ uni-caudal). Além disso, os resultados indicaram que as mesmas características (Honestidade, Fidelidade, Compromisso Sério e Saúde) foram consideradas as mais importantes para ambos os gêneros. Esses resultados não oferecem suporte à previsão da Psicologia Evolucionista, segundo a qual as características mais importantes para um potencial parceiro seriam, respectivamente, para mulheres e homens, status socioeconômico e beleza física. Os dados sugerem que fatores evolutivos e culturais atuam em conjunto influenciando no comportamento de escolha de parceiros para ambos os gêneros.

Palavras-chave: diferenças de gênero, etologia humana, seleção sexual, relacionamento duradouro, comportamento de escolha.

Abstract: This study aimed to perform a comparison between male and female criteria for choosing partners. Attended by 50 men and 50 women students at the University of Rio Verde, who responded to a questionnaire regarding personal data and the characteristics of a hypothetical partner for lasting romantic relationship. There were no statistically significant differences between genders in the importance attributed to the characteristics of potential partners. Furthermore, the results indicated that the same characteristics (Honesty, Loyalty, Commitment and Serious Health) were considered the most important for both genders. These results do not support the prediction of Evolutionary Psychology, according to which the most important characteristics for a potential partner would be, respectively, for women and men, socioeconomic status and physical beauty. The data suggest that evolutionary and cultural factors act together in influencing behavior choice of partners for both genders.

Keywords: gender differences, human ethologic, sexual selection, lasting relationship, mate choice behavior.

\section{Introdução}

Estudos anteriores têm evidenciado a diferença entre os gêneros na escolha de características desejáveis num possível parceiro para relacionamento duradouro (Buss, 1985; Buss \& Schmidt, 1993; Moura, 2005; Varela \& Ferreira, 2006; Altafim, Caramaschi \& Lauandos, 2009). A literatura relacionada à Psicologia Evolutiva enfatiza a investigação dos critérios femininos de parceiros em detrimento dos critérios masculinos (Marquezan, 2005) devido à hipótese de que as mulheres seriam mais seletivas do que os homens em relação a características que poderiam indicar melhores condições para a criação dos filhos. Apesar disso, embora a Psicologia Evolutiva tenha como pressuposto a noção de critérios de escolha de parceiros seriam universais (Buss \& Schmidt, 1993; Oliva, Otta, Ribeiro, Bussab, Lopes, Yamamoto \& Moura, 2006), torna-se necessário investigar esses critérios em diferentes culturas para verificar empiricamente essa afirmativa.

Ao longo da evolução, machos e fêmeas de primatas se diferenciaram quanto às 
características preferidas em seus parceiros através do mecanismo de seleção sexual e da seleção natural (Buss \& Schmidt, 1993; Marquezan, 2005). A quantidade de energia investida na criação dos filhos é diferente para os machos e as fêmeas, pois são elas que fazem um investimento maior, devido à gestação, amamentação, cuidados pós-natais. Como elas tendem a ser mais seletivas, isso acarreta consequência nas estratégias utilizadas para a resolução dos problemas de reprodução (Altafim, Caramaschi \& Lauandos, 2009).

Dessa forma, segundo a Psicologia Evolucionista (Buss \& Schmidt, 1993; Marquezan 2005; Oliva et al., 2006), assim como ocorre com os primatas em geral, os homens têm sido avaliados por seu sucesso econômico e, as mulheres, por sua atratividade física.

Contudo, embora a variação nas preferências de escolha de parceiros entre os gêneros tenha sido atribuída a fatores exclusivamente evolutivos (Buss, 1985; Buss \& Schmidt, 1993; Altafim, Caramaschi \& Lauandos, 2009), outros estudos vêm apresentando resultados que sugerem que fatores biológico e cultural atuam influenciando mutuamente no controle do comportamento de escolha de parceiros em humanos (Nina-e-Silva, Loja, Dominot \& Nascimento-Jr, 2000; Marquezan, 2005).

Por outro lado, segundo Borrione e Lordelo (2005), os relacionamentos de curto prazo possibilitam avaliar os futuros candidatos para um relacionamento de longo prazo. De acordo com esses autores, as pessoas decidem as qualidades que querem e os defeitos que não querem num parceiro amoroso, mas nem sempre possuem muita consciência de que fazem isso. Normalmente, o que se espera nessa procura de parceiro é um parceiro com mais características semelhantes ao próprio indivíduo. Desse modo, os relacionamentos de curto prazo possibilitam a identificação de características desejáveis no possível companheiro para eventuais relacionamentos duradouros.

No estudo de Marquezan (2005), o objetivo foi testar hipóteses ligadas às teorias evolucionistas e de mercado. A amostra foi composta por 200 sujeitos do sexo masculino e 200 do sexo feminino, que responderam a um questionário referente a dados pessoais e às preferências de características que um hipotético parceiro para relacionamento duradouro deveria ter. Os resultados deste estudo corroboraram apenas parcialmente a previsão da Psicologia Evolucionista (Buss, 1985; Buss \& Schmidt, 1993) segundo a qual os homens valorizariam mais atributos de beleza em uma possível parceira, enquanto as mulheres se interessariam mais pelo nível socioeconômico de um possível parceiro. Apesar de os homens terem significativamente atribuído maior importância para a beleza e as mulheres, para o potencial econômico, as características Honestidade e Fidelidade foram consideradas as mais importantes para ambos os gêneros. 
Dessa forma, Marquezan (2005) concluiu que tanto fatores evolutivos quanto de aprendizagem social influenciariam $\mathrm{o}$ comportamento de escolha de parceiros.

Conclusões semelhantes foram apresentadas pela pesquisa de Nina-e-Silva e cols. (2000). Neste estudo, cujo objetivo foi avaliar os critérios femininos de seleção de parceiros em Goiânia, as participantes descreveram e ordenaram atributos de três tipos distintos de hipotéticos parceiros: parceiro romântico ideal; pai ideal e parceiro sexual ideal. Verificou-se que as características consideradas desejáveis pelas mulheres variaram consideravelmente conforme o tipo de parceiro, o que contraria as previsões da Psicologia Evolucionista, segundo a qual as características desejáveis em um parceiro para as mulheres estariam invariavelmente relacionados à capacidade real ou potencial de sustento da prole pelo homem. A partir dessas evidências, Nina-eSilva e cols. (2000) concluíram que os critérios femininos de seleção de parceiros seriam influenciados por múltiplos fatores, evolutivos e culturais, e variariam conforme o contexto de escolha.

Sobre essa hipótese de que o contexto de escolha seria uma variável relevante para a seleção de parceiros, Altafim, Caramaschi e Lauandos (2009) realizaram uma pesquisa para avaliar a diferença entre os gêneros na escolha de características desejáveis num possível companheiro nos contextos diferentes (festa e faculdade). Os resultados indicaram que ambos os sexos são mais seletivos em relação a algumas características na faculdade em comparação à festa.

Em vista disso, homens e mulheres teriam adaptações mentais distintas voltadas tanto para a adoção de estratégias de curto prazo em alguns contextos, quanto para adoção de estratégias de longo prazo em outras situações (Altafim, Caramaschi \& Lauandos, 2009). Essa flexibilidade teria dado aos nossos ancestrais importantes benefícios reprodutivos por permitir que eles respondessem adaptativamente a uma grande variedade de contextos: familiar, cultural e ecológico (Buss, 1985; Marquezan, 2005). Entretanto, reconhece-se que homens e mulheres teriam inclinações e preferências diferentes para cada nível de relacionamento em virtude da aprendizagem social, sendo relevante a análise das normas de escolha de parceiros que a cultura prescreve para cada gênero (Ferreira \& Varella, 2006).

Portanto, para a Psicologia Evolucionista, as diferenças no critério de homens e mulheres seriam estratégias evolutivamente estáveis que foram selecionadas ao acaso em virtude dos diferentes problemas adaptativos que cada sexo enfrentou ao longo de nossa história evolutiva (Buss, 1985; Buss \& Schmidt, 1993; Borrione \& Lordelo, 2005; Oliva e cols., 2006). Os problemas adaptativos femininos estariam relacionados a assegurar recursos para si e para sua prole. Já os problemas masculinos estariam relacionados à 
certeza da fertilidade da parceira e a assegurar paternidade por meio do controle de acesso sexual à parceira.

Desse modo, os objetivos do presente estudo foram: 1) comparar os critérios masculino e feminino de escolha de parceiros para um relacionamento afetivo duradouro; 2) replicar parcialmente o estudo de Marchezan (2005), identificando o grau de importância atribuído por homens e mulheres a características de um hipotético parceiro para relacionamento duradouro; e 3) levantar as características consideradas mais desejáveis por homens e mulheres em um possível parceiro para relacionamento duradouro.

\section{Materiais e Métodos}

\subsection{Participantes}

Cem estudantes universitários (50 homens e 50 mulheres), provenientes de cursos variados da Universidade de Rio Verde

- UNIRV, dispuseram-se a participar da pesquisa e assinaram o Termo de Consentimento Livre e Esclarecido.

\subsection{Instrumento}

Para a avaliação do grau de importância de características de prováveis parceiros, os participantes responderam ao Questionário 1 (Marchezan, 2005) que apresentou 19 características (Anexo 2). Os participantes atribuíram a cada característica uma nota que poderia variar de 1 a 10, indicando em ordem crescente da nota o grau de importância que davam à característica em um parceiro para relacionamento estável. Em seguida, os participantes responderam ao Questionário 2 (Nina-e-Silva e cols., 2000), citando, em ordem de importância, três características indispensáveis em um hipotético parceiro para relacionamento duradouro.

\subsection{Procedimentos}

Todos os instrumentos de coleta de dados e o procedimento de aplicação foram previamente submetidos à aprovação do Comitê de Ética em Pesquisa da Universidade de Rio Verde (protocolo 058/2010).

Os participantes foram abordados em diferentes locais da Universidade: cantina, biblioteca, corredor e salas de aula para serem convidados a participar da pesquisa. Depois da leitura do TCLE e do esclarecimento de eventuais dúvidas, assinaram o TCLE e, em seguida, receberam os questionários com a instrução de respondê-los, posteriormente, em casa ou em qualquer outro local em que se sentissem confortáveis. Combinou-se com cada participante local, data e horário para o recolhimento dos questionários preenchidos.

\subsection{Análise de Dados}

A avaliação do grau de importância atribuído por homens e mulheres a características de um hipotético parceiro para relacionamento duradouro foi feita por meio da comparação das médias de notas atribuídas pelos participantes a cada uma das características no Questionário 1. A verificação da existência de diferenças 
significativas entre os gêneros nas atribuições de notas às características foi realizada através do teste $\mathrm{t}$ de diferenças de média sem que fossem assumidas igualdade de variâncias entre as amostras.

O levantamento das características consideradas mais desejáveis por homens e mulheres em um possível parceiro para relacionamento duradouro foi feito por meio do cálculo da ordem média de citação (OMC) das características evocadas pelo Questionário 2. Cada característica recebeu um peso correspondente à ordem de citação de cada participante. Desse modo, uma característica citada em primeiro lugar por um participante recebeu um peso de valor 1 , enquanto que uma característica citada em segundo lugar recebeu um peso 2, e assim por diante. Para cada característica, realizou-se o somatório dos pesos recebidos, o qual foi dividido pelo número total de citações dessa característica, obtendo-se desse modo a OMC. Quanto menor o valor da OMC calculado para uma característica, considerou-se mais desejável essa característica para o grupo estudado. A frequência de citações de cada característica foi levada em conta na análise para evitar eventuais distorções na ordenação por grupo (no nosso caso, gênero) das características resultantes do uso apenas da OMC.

\section{Resultados e Discussão}

A análise dos dados obtidos no Questionário 1 indicou que não houve diferença estatisticamente significativa entre as médias de notas atribuídas por homens e mulheres às características de um hipotético parceiro para relacionamento afetivo duradouro ( $t=1,24 ; p=0,12$ uni-caudal).

A comparação das médias de notas atribuídas pelos participantes a cada uma das características na questão 1 mostrou que não houve diferenças significativas entre os gêneros: Ambicioso (a) $(t=0,44 ; p=0,32)$, Atlético (a) ( $t=0,83 ; p=0,21)$, Satisfação Sexual $(t=0,04 ; p=0,49)$, Bonito (a) $(t=1,36$; $p=0,10)$, Criativo (a) $(t=-0,28 ; \mathrm{p}=0,39)$, Emocionalmente Estável $(t=0,38 ; p=0,36)$, Escolaridade $(t=0,72 ; p=0,24)$, Habilidades Domésticas $(t=0,78 ; p=0,22)$, Bem Humorado (a) $(t=-0,34 ; p=0,37)$, Bom Emprego $(t=-0,36 ; p=0,36)$, Deseja Ter Filhos $(t=-0,28 ; p=0,39)$, Dinheiro $(t=0,09$; $p=0,46)$, Inteligente $(t=0,81 ; p=0,22)$, Religioso (a) $\quad(t=\quad-0,07 ; \quad p=0,47)$ Compreensivo (a) ( $t=-0,17 ; \quad p=0,43)$, Compromisso Sério $(t=-0,42 ; p=0,34)$, Fiel $(t=0,21 ; p=0,42)$, Honesto (a) $(t=0,21 ; p=$ $0,42)$, Saudável $(t=0,04 ; p=0,48)$.

As características foram classificadas em posições de acordo com a ordem decrescente de valor de média (Tabela 1). De modo geral, não houve variações quanto às classificações dos atributos escolhidos pelos homens e mulheres na escolha de um parceiro para um relacionamento duradouro.

Verificou-se que as quatro primeiras posições para ambos os gêneros foram ocupadas, respectivamente, pelas mesmas características: $\quad$ Fiel, Honesto(a), 
Compromisso Sério e Saudável. A variação máxima de posição de características observada entre os gêneros foi de cinco postos. As características que apresentaram essa variação máxima foram Bem Humorado(a); Bonito(a) e Religioso(a). Todas as demais características apresentaram variação de posição igual ou abaixo de quatro postos entre os gêneros.

Homens e mulheres atribuíram nota máxima (dez) no Questionário 1 às mesmas características, com exceção de Bem Humorado, Escolaridade, Bom Emprego,
Religioso e Habilidades Domésticas, atributos pontuados apenas pelas mulheres (vide Tabela 2).

A análise dos dados do Questionário 2 indicou que, de modo geral, homens e mulheres citaram as mesmas características consideradas desejáveis em um hipotético parceiro para um relacionamento duradouro. As únicas exceções foram as características Respeito e Dedicação (citadas uma única vez apenas por mulheres) e as características Carisma e Paixão (citadas uma única vez apenas por homens).

TABELA 1: Disposição em ordem decrescente das médias atribuídas pelos participantes homens e mulheres às características de um hipotético parceiro para relacionamento afetivo duradouro no Questionário 1.

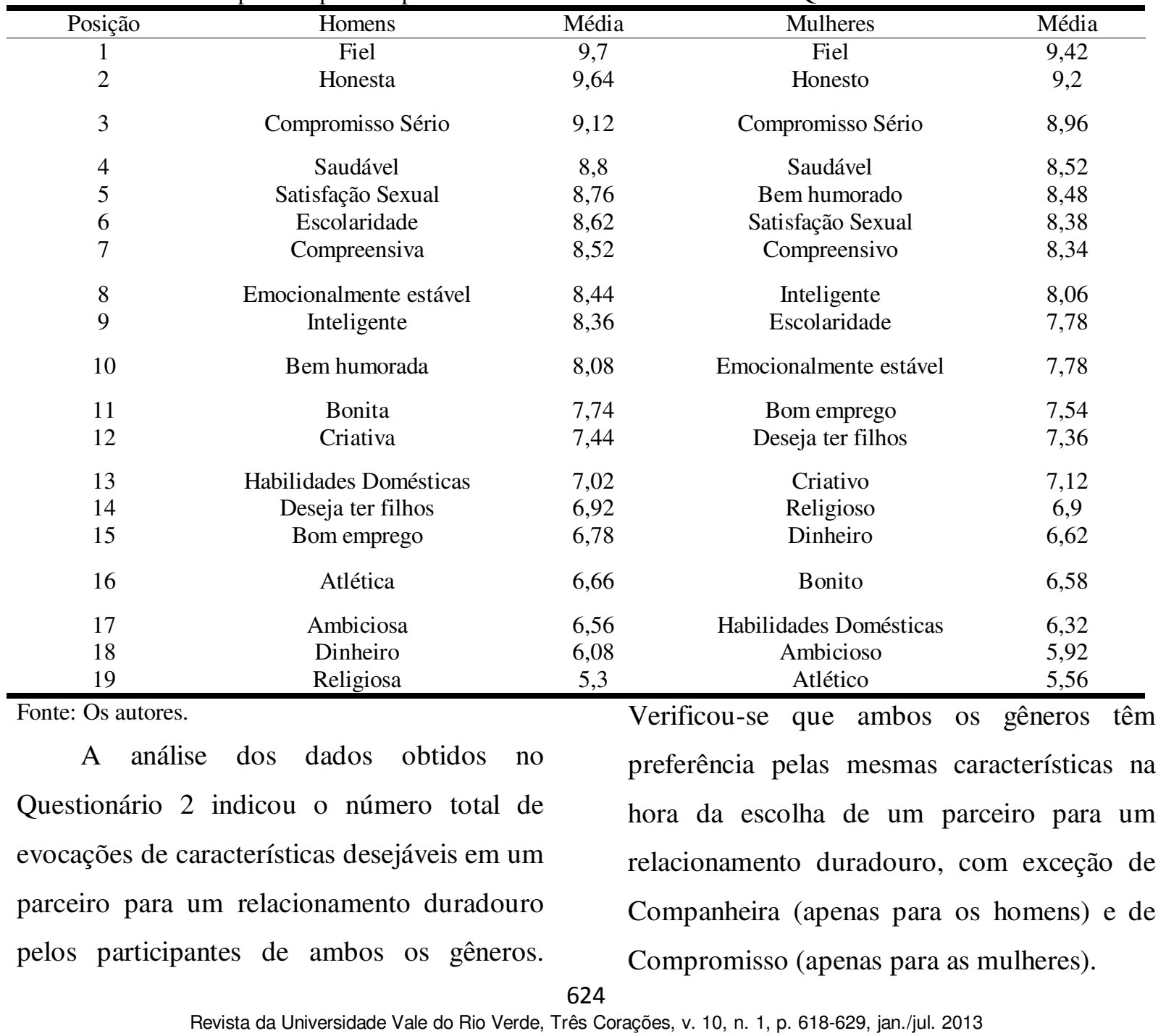


Com relação à posição de importância da evocação das características, verificou-se que ambos os gêneros citaram as características Fidelidade e Honestidade, respectivamente, nas posições de importância 1 e 2 . As características que apresentaram as maiores diferenças entre os gêneros quanto à posição de importância da evocação foram:

Compreensivo (homens=5; mulheres=3) e inteligente (homens=3; mulheres $=5$ ).

De modo geral, os resultados do presente estudo não corroboram o ponto de vista da Psicologia Evolucionista (Marquezan 2005; Borrione e Lordelo, 2005; Oliva e cols.,
2006) segundo o qual homens e mulheres apresentariam critérios significativamente diferentes de escolha de parceiros.

Os resultados de Marquezan (2005) evidenciaram diferenças estatisticamente significativas entre os gêneros na atribuição de pontuação às 19 características listadas no Questionário 1. Contudo, no presente estudo, não houve diferenças estatisticamente significativas entre os gêneros na atribuição de notas às características.

TABELA 2: Características que receberam pontuação máxima no Questionário 1 para cada um dos gêneros.

\begin{tabular}{ll}
\hline Homens & Mulheres \\
\hline Fiel & Fiel \\
Honesta & Honesto \\
Sério & Compromisso \\
Satisfação Sexual & Satisfação Sexual \\
Saudável & Saudável \\
Compreensiva & Compreensivo \\
Deseja ter filhos & Deseja ter Filhos \\
& Bem Humorado \\
& Escolaridade \\
& Bom Emprego \\
& Religioso \\
& Habilidades Domésticas \\
\hline
\end{tabular}

Fonte: Os autores.

TABELA 3: Disposição em ordem decrescente do número total de evocações de características desejáveis em um parceiro para relacionamento duradouro pelos participantes de ambos os gêneros.

\begin{tabular}{clcclcc}
\hline & & \multicolumn{2}{c}{ GÊNERO } & \\
POSIÇÃO & \multicolumn{1}{c}{ HOMENS } & $F$ & OMC* & \multicolumn{1}{c}{ MULHERES } & $f$ & OMC* $^{*}$ \\
\hline 1 & FIEL & 34 & 1,50 & FIEL & 31 & 1,77 \\
2 & HONESTA & 25 & 2,20 & HONESTO & 25 & 1,64 \\
3 & INTELIGENTE & 20 & 1,90 & COMPREENSIVO & 17 & 2,10 \\
4 & COMPANHEIRA & 11 & 1,11 & COMPROMISSO & 14 & 2,10 \\
5 & COMPREENSIVA & 10 & 2,60 & INTELIGENTE & 11 & 1,45 \\
\hline
\end{tabular}

*Ordem média de citação.

Fonte: Os autores. 
No estudo de Marquezan (2005), os homens atribuíram notas significativamente mais altas do que as mulheres às características Beleza e Atlética. Esses dados estão de acordo com a previsão da Psicologia Evolucionista (Borrione e Lordelo, 2005; Oliva e cols., 2006) segundo a qual os homens se sentiriam mais atraídos pelas características que demonstram bom potencial de reprodução e saúde, tais como a Beleza e a Atlética. Por outro lado, contrariando a previsão da Psicologia Evolucionista para a preferência masculina por beleza e saúde em uma hipotética parceiro, não houve atribuição de notas significativamente mais altas pelos homens a nenhuma das características listadas no presente estudo.

Marquezan (2005) descreveu que as mulheres atribuíram notas significativamente mais altas do que os homens para as características: Compromisso, Dinheiro, Bom Emprego e Religiosidade. Esses resultados encontram suporte na literatura relacionada à Psicologia Evolucionista (Borrione e Lordelo, 2005; Oliva e cols., 2006) de acordo com a qual as mulheres tenderiam a selecionar os seus parceiros com base nos recursos que ele poderiam oferecer a elas e à prole. No entanto, os resultados do presente trabalho indicaram que não houve atribuição de notas significativamente mais altas pelas mulheres a nenhuma das características apresentadas no Questionário 1.

Os fatores biológicos e culturais se influenciam reciprocamente no estabelecimento de preferências de escolha de parceiros sexuais. De acordo com a Psicologia Evolucionista, essas preferências se diferenciaram entre os gêneros durante o processo de seleção natural.

Já a Psicologia Social investe na possibilidade de que os estereótipos culturalmente instituídos podem servir de base para as diferenças de gênero na escolha de parceiros (Marquezan, 2005). A partir disso, pode-se entender que as mulheres e os homens realmente são seletivos na hora de selecionar um parceiro e quê, como prevê a literatura, as mulheres preferem parceiros que sejam fiéis e honestos. Mas um ponto diferente da literatura é que esta também são as características que os homens consideram como importantes na escolha de uma parceiro.

De acordo com Altafim, Caramaschi e Lauandos (2009), as mulheres são mais criteriosas ao escolherem parceiros para qualquer nível de envolvimento. Enquanto os homens não considerariam essas características importantes, dando preferência a atributos físicos. Os resultados do presente não dão suporte a essa afirmação. As quatro características que receberam as notas mais altas tanto por homens quanto por mulheres foram exatamente as mesmas: Fidelidade, Honestidade, Compromisso e Saúde (Tabela 1). Honestidade e Fidelidade também foram as duas características com as maiores notas atribuídas pelos participantes de ambos os gêneros do estudo de Marquezan (2005). 
As características Beleza e Atléticas, consideradas as mais relevantes para os homens pela Psicologia Evolucionista (Oliva e cols., 2006), ficaram, respectivamente, na $11^{\mathrm{a}}$ e na $16^{\mathrm{a}}$ posições. O estudo de Marquezan (2005) descreveu resultados semelhantes aos nossos, visto que as características Beleza e Atlética ficaram, respectivamente, na $12^{\mathrm{a}}$ e na $13^{\mathrm{a}}$ posições.

No estudo de Marquezan (2005), as mulheres consideraram como características mais relevantes na escolha de um parceiro os quesitos "compromisso", "nível socioeconômico", “bom emprego" e "religiosidade". Resultados semelhantes foram encontrados por nosso estudo, pois as mulheres evidenciaram interesse por um parceiro que fosse "fiel", "honesto" e "queira um compromisso sério", ficando essas características, respectivamente nas 3 primeiras posições.

Os resultados que indicaram que os homens buscam parceiras que sejam fiéis, e honestas, para se ter um relacionamento duradouro estão de acordo com a literatura (Altafim, Caramaschi \& Lauandos, 2009). Segundo esses autores, a mulher que mantém longos relacionamentos seria mais respeitada e valorizada pelos homens do que aquela que se relaciona por um curto período de tempo.

Os resultados do Questionário 2 encontram suporte parcial no estudo de Ninae-Silva e cols. (2000), pois em ambos os trabalhos as características Honesto e Inteligente estiveram entre as cinco características mais frequentemente citadas pelas mulheres. No entanto, a característica Bonito, que aparece entre as cinco mais frequentemente citadas pelas mulheres em Nina-e-Silva e cols. (2000), foi pouco citada pelas participantes do presente estudo.

De acordo com a literatura evolucionista (Borrione e Lordelo, 2005; Marchezan, 2005; Oliva e cols., 2006), as mulheres são mais exigentes na escolha de parceiros do que os homens. Isso acontece porque a mulher tem um investimento biológico muito maior do que o homem em uma eventual gravidez. Dessa forma, a mulher tenderia a ser mais seletiva na escolha de seus parceiros para assegurar sustento para si e para sua prole (Borrione e Lordelo, 2005).

No entanto, os resultados gerais do presente estudo não corroboram esse ponto de vista evolucionista, visto que tanto os homens quanto as mulheres demonstraram grau parecido de exigência em relação à pontuação atribuída às características preferidas na hora da escolha de parceiros.

\section{Conclusão}

O presente trabalho teve como objetivo geral comparar os critérios masculino e feminino de escolha de parceiro para um relacionamento afetivo duradouro. Os resultados indicaram que não houve diferença significativa entre os critérios masculino e feminino de seleção de parceiros.

Este trabalho também replicou, parcialmente, o estudo de Marchezan (2005), 
identificando que as características consideradas mais desejáveis por homens e mulheres em um possível parceiro para um relacionamento duradouro são: Fiel, Honesto e Que queira um compromisso sério. Além disso, esta pesquisa avaliou o grau de importância atribuído por homens e mulheres a características de um hipotético parceiro para um relacionamento duradouro e verificou que os gêneros atribuem graus de importância semelhantes a praticamente às mesmas características.

Dessa forma, os resultados gerais contrariam as hipóteses exclusivamente psicobiológicas da Psicologia Evolucionista (Buss, 1985; Borrione \& Lordelo, 2005; Oliva e cols., 2006) e fortalecem o pressuposto de multideterminação do comportamento de escolha de parceiros (Nina-e-Silva e cols., 2000; Marquezan, 2005).

\section{Referências Bibliográficas}

ALTAFIM, E. R. P.; CARAMASCHI, S.;

LAUANDOS, J. M. Seleção de parceiros: diferenças entre gêneros em diferentes contextos. Psicologia Argumentativa, v. 27, n. 57 , p. 117-129, 2009.

BUSS, D.M. Human mate selection.

American Scientist, 73, 47-51, 1985.

BUSS, D.M. \& SCHMIDT, D.P. Sexual strategies theory: an evolutionary perspective on human mating. Psychological Review,
MARQUEZAN, R. F. Diferenças entre

gêneros nas preferências para escolha de parceiros em função de fatores biológicos e sociais. Dissertação apresentada ao departamento de psicologia da Universidade Católica de Goiás, para obtenção do título de Mestre em Psicologia, 2005.

MOURA, M. L. S. Dentro e Fora da Caixa Preta: A Mente sob um Olhar Evolucionista.

Psicologia: Teoria e Pesquisa. 21(2) 141147, 2005.

NINA-E-SILVA, C. H.; LOJA, B.O.B; DOMINOT, A.; \& NASCIMENTO-JR, L.C. Critérios femininos de escolha de parceiros em Goiânia: avaliação sociobiológica e ambientalista. Anais de Etologia.

Florianópolis: Sociedade Brasileira de Etologia. p.150, 2000.

OLIVA, A. D.; OTTA, E.; RIBEIRO, F. L.; BUSSAB, V. S. R.; LOPES, F. A.; YAMAMOTO, M. E. \& MOURA, M. L. S. Razão, Emoção e Ação em Cena: A Mente Humana sob um Olhar Evolucionista.

Psicologia: Teoria e Pesquisa. 22 (1) 053062, 2006.

SANTOS, L. L. G. Auto-avaliação da atratividade e os critérios femininos de escolha de parceiros. Monografia apresentada à Faculdade de Psicologia da Universidade de Rio Verde - UNIRV, como parte das 100, 204-232, 1993. 
exigências para obtenção do título de

Psicóloga, 2010.

VARELLA, M. A. C. \& FERREIRA, J. H. B.

P. Sexo Casual: a falta de envolvimento

afetivo é estudada sob a abordagem evolutiva.

Psique Ciência \& Vida. II, 18, 80 - 81,

2006. 